\title{
Mitteilungen des BV Geriatrie
}

Z Gerontol Geriat 2020 · 53:481-484 https://doi.org/10.1007/s00391-020-

01760-z

(c) Springer Medizin Verlag $\mathrm{GmbH}$, ein

Teil von Springer Nature 2020
Geschäftsstelle

Bundesverband Geriatrie e.V.

Reinickendorfer Straße 61

13347 Berlin

Tel.: $\quad$ (030) 339887610

Fax: (030) 339887620

geschaeftsstelle@bv-geriatrie.de

www.bv-geriatrie.de
Vorstandsvorsitzender

Dipl. Kfm. Ansgar Veer

Geschäftsführer

St. Bonifatius Hospital Lingen/Ems

ansgar.veer@bv-geriatrie.de

Geschäftsführer

RA Dirk van den Heuvel (v.i. S. d.P.)

dirk.van-den-heuvel@bv-geriatrie.de
Liebe Mitglieder,

sehr geehrte Damen und Herren,

diese Zeilen entstehen Ende Juni - und damit in einer Zeit, in der zunehmend eine gefühlte „Corona-Normalität" einkehrt und sich gleichzeitig immer wieder neue Corona-Hotspots bilden. Diese Ausbrüche verdeutlichen, dass die Pandemie noch nicht vorbei und das Virus aus dem Alltag verschwunden ist. Gleichwohl treten nach und nach vermehrt andere Themen zurück auf die gesundheitspolitische Agenda. So geht unter anderem das Gesetzgebungsverfahren zum Intensivpflegeund Rehabilitationsstärkungsgesetz (GKV-IPReG) weiter. Dabei steht auch weiterhin der erste Gesetzesteil im Mittelpunkt der Diskussion, in dem insbesondere die Versorgung von Langzeitbeatmungspatienten neu geregelt wird. Diese Neuregelung stößt auf breiten Widerspruch bei den Betroffenen. Dies ist auch der Grund, warum sich das Gesetzgebungsverfahren sehr in die Länge zieht und die wichtigen Regelungen für die Rehabilitation, die im zweiten Gesetzesteil normiert sind, leider weiterhin auf sich warten lassen. Bei der Anhörung im Bundestag Mitte Juni war der Bundesverband Geriatrie als Experte geladen. Es zeigte sich, dass die im Gesetz enthaltenen speziellen Regelungen zur geriatrischen Rehabilitation von anderen Verbänden kritisch bewertet werden. Dabei ist der inhaltliche Regelungsgehalt nicht umstritten, man möchte diese Regelungen jedoch auf den jeweils eigenen Rehabilitationsbereich adaptieren. Dabei geht es insbesondere um die "genehmigungsfreie“ Zuweisung von Patienten durch niedergelassene Ärzte in Rehabilitationskliniken. Coronabedingt wurde die Anhörung im Bundestag ausschließlich digital abgehalten, was die Erörterung der Themen - auch durch technische Probleme - nicht unbedingt erleichterte.

Wie der Bundestag arbeitet auch das Bundesministerium für Gesundheit noch stark im „Coronamodus“, d.h. sowohl thematisch als auch organisatorisch bestimmt dieses Thema sehr stark die Arbeit der Mitarbeiter. Organisatorisch bedeutet dabei, dass ein Großteil der Mitarbeiter noch im Homeoffice tätig ist. Vor diesem Hintergrund fand auch der jüngste Austausch mit dem Staatssekretär Herrn Dr. Gebhart leider nur als Telefonkonferenz statt. Diese technischen Rahmenbedingungen erleichterten nicht unbedingt den Aufbau einer persönlichen Gesprächsatmosphäre in der ein vertrauliches Hintergrundgespräch geführt werden konnte. Letztlich konnte diese „Hürde“ jedoch genommen werden und es fand ein intensiver Austausch statt. Dieser Dialog soll im Laufe der nächsten Monate fortgeführt werden.

Der Gesprächszeitpunkt war sehr gut gewählt, da aktuell viele Gesetze und Verordnungen, die zu Beginn der Coronakrise - zum Teil in Rekordgeschwindigkeit - erarbeitet und erlassen wurden derzeit überarbeitet, ergänzt bzw. an die aktuelle Situation angepasst werden. Auch in diesem Prozess ist der Bundesverband Geriatrie direkt und über sein Netzwerk intensiv beteiligt und bringt die Interessen der Geriatrie entsprechend ein. Leider findet dieser Überarbeitungsprozess teilweise mit einer ähnlich hohen Geschwindigkeit wie in der akuten
Phase der Pandemie statt, sodass zumeist nur sehr wenig Zeit sowohl für die offiziellen Stellungnahmen als auch für gezielte Hintergrundarbeit bleibt. Es bleibt abzuwarten, ob dieser Trend auch über die Sommermonate anhält oder diese Zeit für verstärkte inhaltliche Gesetzesarbeit genutzt wird.

Ihr

Dirk van den Heuvel

\section{Gespräch mit dem Staatssekretär Dr. Thomas Gebhart}

Wie kann die medizinische Versorgung geriatrischer Patienten zukünftig sachgerecht finanziert werden? Diese Frage war das dominierende Thema bei einem Gespräch zwischen dem Bundesministerium für Gesundheit (BMG) und dem BV Geriatrie. Dr. Thomas Gebhart, Parlamentarischer Staatssekretär beim Bundesminister für Gesundheit (BMG), sowie Ansgar Veer, Vorstandsvorsitzender des BV Geriatrie, und Dirk van den Heuvel, Geschäftsführer des BV Geriatrie, eröffneten den Dialog, bei dem das Finanzierungsmodell „Besondere Einrichtungen" als alternative Finanzierungsform im Fokus stand. Durch die Ausgliederung der Pflegepersonalkosten beziehungsweise die Einführung des Pflegebudgets gewinnen alternative Finanzierungsformen im Krankenhausbereich immer mehr an Bedeutung. Im Bereich der Rehabilitation war die auskömmliche Gestaltung der Tagessätze die zentrale Frage.

Des Weiteren standen Themen wie etwa das Intensivpflege- und Rehabilitationsstärkungsgesetz (GKV-IPReG) sowie die perspektivische Weiterentwicklung der geriatriespezifischen Versorgungslandschaft auf der Agenda. Unter anderem wurden die Herausforderungen aufgrund von Umstrukturierungen im Krankenhausbereich durch die COVID-19-Pandemie aufgezeigt, die Wichtigkeit eines weiteren Ausbaus der sektorenübergreifenden Versorgung insbesondere im Bereich der Geriatrie deutlich gemacht und die Umsetzung des bundesweiten Geriatriekonzeptes in die Praxis erörtert.

Ziel des Austauschs ist es, die Geriatrie auch weiterhin im Fokus des BMG zu halten und bei den zentralen Themen Finanzierung und Weiterentwicklung der Versorgungsstrukturen die Unterstützung des Ministeriums zu sichern. Coronabedingt fand das Gespräch, bei dem auch mehrere Fachvertreter des Ministeriums beteiligt waren, Anfang Juni als Telefonkonferenz statt. 


\section{Kodierhandbuch Geriatrie 2020 im Handel erhältlich}

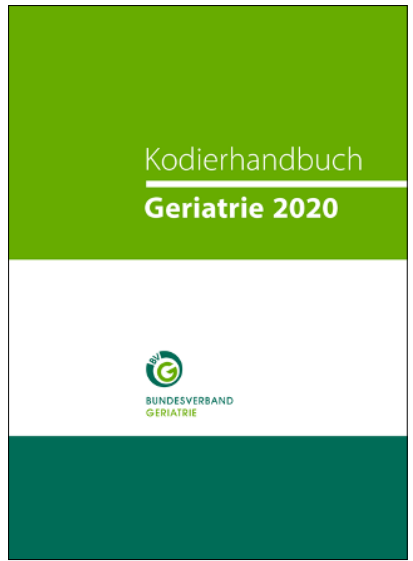

$\Delta$ Herausgeber: Bundesverband Geriatrie, Seiten: 382, Ausstattung: Softcover, Auflage: 11. überarbeitete Auflage 2020, Verlag: Schüling-Verlag, Münster, ISBN: 978-3-86523-305-9, Preis: 19,80€
Ende Juli 2020 wurde die 11. Auflage des Kodierhandbuches Geriatrie veröffentlicht. Das Kodierhandbuch Geriatrie 2020 wurde mit Blick auf die Änderungen der ICD-10-GM, der Operationen- und Prozedurenschlüssel (OPS) sowie der Deutschen Kodierrichtlinien aktualisiert. Auch in dieser Ausgabe werden die wichtigsten Kodierregeln unter Berücksichtigung geriatriespezifischer Probleme erläutert sowie allgemeine und spezifische Dokumentationshinweise gegeben. In diesem Jahr möchten wir Sie insbesondere auf die umfassend aktualisierten Auslegungshinweise des BV Geriatrie zu den OPS 8-550 ff. und 8-98a ff. hinweisen. Um die Übersichten der Diagnose- und Prozedurenschlüssel präziser zu definieren, ist für die 11. Auflage des Kodierhandbuches zusätzlich eine umfassende Aktualisierung der Hinweise, Inklusiva und Exklusiva erfolgt. Das leistungsrechtliche Kapitel befasst sich für das Jahr 2020 intensiv mit der Ausgliederung der Pflegepersonalkosten im Zuge des Pflegepersonal-Stärkungsgesetzes und den resultierenden Auswirkungen auf die Klassifikation des aG-DRGSystems. Des Weiteren enthält das Kapitel umfassende Ausführungen zum MDK-Reformgesetz sowie zur Pflegepersonaluntergrenzen-Verordnung. Abschließend finden auch die Neureglungen im Rahmen der Corona-Krise Beachtung.

Nachdem das Buch mehrere Jahre unter der Federführung von Frau Wittrich erarbeitet wurde, ist die neue Auflage unter Verantwortung von Frau Kappes entstanden, die - wie berichtet - Anfang des Jahres als Bereichsleiterin Finanzierung die Aufgabenbereiche von Frau Wittrich übernommen hat. Darüber hinaus hat Herr Holtmann, Bereichsleiter Grundsatzfragen beim BV Geriatrie, intensiv an der Neuauflage mitgewirkt.

Das Kodierhandbuch Geriatrie 2020 ist wie in den letzten Jahren direkt über den Buchhandel sowie über die entsprechenden Onlineportale erhältlich.

\section{Patientendaten-Schutzgesetz: Medizinische Rehabilitation außen vor}

Das Ziel des Entwurfes der Bundesregierung eines Gesetzes zum Schutz elektronischer Patientendaten in der Telematikinfrastruktur (Patientendaten-Schutzgesetz - PDSG) vom 27. April 2020 und die diesem zugrunde liegende Intention der Bundesregierung, eine sichere, vertrauensvolle, nutzerfreundliche sowie barrierefreie digitale Kommunikation zwischen Leistungserbringern untereinander und zwischen Leistungserbringern und Patienten zu schaffen sowie auszuweiten, werden vom BV Geriatrie ausdrücklich begrüßt. Dies betrifft insbesondere den vorgesehenen Anschluss weiterer Leistungserbringergruppen an die bereits bestehende Telematikinfrastruktur.

Zwingend erforderlich ist es aus Sicht des BV Geriatrie, die Spitzenverbände der Rehabilitationseinrichtungen in die weitere Entwicklung einer solchen Telematikinfrastruktur einzubeziehen. Zugleich muss es oberste Prämisse sein, patientenindividuelle und sensible Daten adäquat zu schützen und zu sichern.

Der BV Geriatrie fordert eine konsequente Beteiligung der Vorsorge- und Rehabilitationseinrichtungen. So sollen unter anderem Vertreter der Spitzenverbände der Rehabilitations-Leistungserbringer in den Beirat der Gesellschaft für Telematik berufen werden. Und auch bei für die medizinische Rehabilitation relevanten Fragestellungen sollen die für die Vorsorge- und Rehabilitationseinrichtungen maßgeblichen Spitzenverbände Mitspracherecht erhalten.

Die vollständige Stellungnahme des BV Geriatrie zum PDSG steht unter folgendem Link zum Download bereit: https://bit.ly/3c3GsUj

\section{Facharzt Innere Medizin und Geriatrie bleibt in Brandenburg bestehen}

Gute Nachrichten aus Brandenburg: Nachdem es Überlegungen der Ärztekammer in Brandenburg gab, die Weiterbildungsordnung im Bereich Innere Medizin bzw. Geriatrie zu ändern, hat die Kammer am 20. Juni 2020 einstimmig entschieden, dass der FA Innere Medizin und Geriatrie in Brandenburg auch weiterhin bestehen bleibt. Der Landesverband Geriatrie Brandenburg hatte sich im Vorfeld der Kammerentscheidung intensiv für den Erhalt des Facharztes eingesetzt und viele Gespräche dazu geführt. Entsprechend positiv hat man die deutliche Entscheidung begrüßt.

\section{Nachgefragt: Wie läuft es bei TIGER?}

Das Projekt TIGER (Transsektorales Interventionsprogramm zur Verbesserung der geriatrischen Versorgung in Regensburg) startete im Jahr 2017. Es wurde von Prof. Dr. Cornel Sieber und PD Dr. Ellen Freiberger vom Institut für Biomedizin des Alterns (IBA) der FriedrichAlexander-Universität Erlangen-Nürnberg initiiert. Der BV Geriatrie ist als Konsortialpartner des Projektes an den ersten Arbeitspaketen sowie auch in der letzten Phase des Forschungsprojektes, im Jahr 2020, maßgeblich bei der Etablierung des transsektoralen Ansatzes in die Versorgungslandschaft beteiligt.

Intention des TIGER-Projekts ist es, mit Hilfe von sogenannten „Pfadfindern“ den Übergang vom Krankenhaus zur ambulanten Ver- 


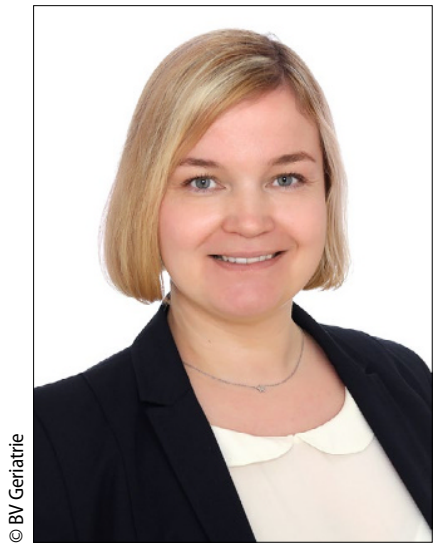

$\Delta$ Nadine Morkisch ist wissenschaftliche Mitarbeiterin des BV Geriatrie sorgung vorzubereiten sowie zu begleiten. Pfadfinder sind geriatriespezifisch ausgebildete Pflegefachpersonen. Sie beraten Patienten und Angehörige und bereiten schon während des Krankenhausaufenthaltes die Zeit nach der Entlassung vor, wie etwa die Koordination von Hilfen und/ oder Hilfsmitteln. Die Pfadfinder erfassen Risiken und Symptome der Patienten und unterstützen sie bei der Verbesserung der Kompetenzen im Selbstmanagement. Dabei stimmen sie sämtliche Maßnahmen mit den Patienten, deren Angehörigen, Ärzten und Pflegepersonal ab.

Dieser neue Versorgungsansatz wird in einer randomisierten kontrollierten Studie evaluiert. Erhoben werden unter anderem Daten zur Wiederaufnahmerate. Ist das Konzept erfolgreich, kann mit Hilfe der Pfadfinder die Häufigkeit von Wiederaufnahmen gesenkt und die Selbstständigkeit und Lebensqualität der Patienten erhöht werden.

Im vergangenen Jahr wurden die Ergebnisse des systematischen Reviews präsentiert. Seitdem ist viel passiert. Nadine Morkisch, wissenschaftliche Mitarbeiterin des BV Geriatrie, gibt einen Einblick ihrer aktuellen Arbeiten am TIGER-Projekt.

Frau Morkisch, lassen Sie die vergangenen drei Jahre Revue passieren. Welche wichtigen Meilensteine kennzeichnen TIGER aus Ihrer Sicht?

Wir erhielten sowohl einen internationalen als auch einen bundesweiten Überblick über die Begleitung von geriatrischen Patienten, die sich im Übergang vom Krankenhaus nach Hause befinden. Dabei haben wir die neun Komponenten des amerikanischen „Transitional Care“ Modells (TCM) näher kennengelernt und deren Einsatz untersucht.

Der allgemeine Blick auf die internationale Studienlage zeigte, dass die Idee den Übergang vom Krankenhaus nach Hause zu begleiten bereits vielfach untersucht wurde. Hierbei lag der Fokus auf unterschiedlichen Schwerpunkten, wie zum Beispiel auf verschiedenen Altersgruppen oder Diagnosen beziehungsweise Diagnosegruppen. Auch die Begleitung durch verschiedene Berufsgruppen oder durch ein multidisziplinäres Team sowie verschiedene Modelle, die zum Teil auch technische Lösungen nutzten, wurden evaluiert.

In Bezug auf Personen im Alter über 65 Jahre sind jedoch eher wenige Studien veröffentlicht worden. Dies zeigte eine systematische Literatursuche von 1994 bis zum Jahresende 2019 auf. Anhand von drei eingeschlossenen randomisierten kontrollierten Studien konnte verdeutlicht werden, dass die Anwendung der Komponenten des TCM bei geriatrischen Patienten während des Übergangs vom Krankenhaus nach Hause die Krankenhauswiederaufnahmerate senken kann. Dabei sind ein hohes Intensitätslevel und die Begleitung durch ein multiprofessionelles Team von Vorteil. Als limitierend muss die geringe Studienzahl genannt werden, die wir auf die Kombination der eingesetzten Ein- und Ausschlusskriterien zurückführen.

In den vergangenen Monaten haben Sie Informationen zur aktuellen nationalen Versorgungssituation gesammelt. Was war die Zielstellung und wie sind Sie dabei vorgegangen?

Uns interessierte hierbei, welche Komponenten des TCM in Projekten, Konzepten oder Versorgungsansätzen in Deutschland existie- ren, bei denen geriatrische Patienten vom Krankenhaus in die eigene Häuslichkeit begleitet werden. Darüber hinaus wollten wir etwas über die Gestaltung der Begleitung erfahren.

Hierfür hatten wir Kontaktdaten von Personen aus dem (ehemaligen) Mitgliederkreis des BV Geriatrie gesammelt und mittels Internetrecherche weitere Ansprechpartner, die im Bereich Geriatrie tätig sind, identifiziert. Zusätzlich wurden Krankenkassen, Bundesministerien mit inhaltlichem Bezug und Ministerien für Gesundheit auf Landesebene einbezogen.

In einem Anschreiben stellten wir das Innovationsfondsprojekt TIGER und die Ziele der Umfrage dar. Hatten die angeschriebenen Personen Kenntnis von transsektoralen Projekten im geriatriespezifischen Bereich, so baten wir sie einen Fragebogen auszufüllen.

\section{Was lief gut und bei welchen Projektschritten galt es, Herausforderun- gen zu meistern?}

An dieser Stelle danken wir allen Teilnehmenden für ihr Interesse, ihre freundlichen Rückmeldungen und Ihre Informationen. Die Durchführung der Umfrage mittels Online-Fragebogen verlief sehr gut. Hier möchten wir unseren Kollegen unseres Konsortialpartners des Instituts für Biomedizin des Alterns, für die zur Verfügung gestellte Nutzung der Umfragesoftware danken.

Wir konnten zwar eine gute Rücklaufquote bezogen auf die Personen, die einen Fragebogen angefordert und dann auch beendet hatten erzielen. Jedoch können wir dem Anspruch auf Vollständigkeit nicht genüge tragen, da uns einige Projekte bekannt sind, die nicht näher durch einen Fragebogen beschrieben wurden.

Der geplante Workshop mit interessierten Umfrageteilnehmern musste in den September verschoben werden. Wir sind optimistisch, dass diese Expertengruppe sinnvolle Ergebnisse erarbeiten wird, um die Entwicklung in Richtung einer Übertragung in die Versorgungspraxis zu fördern.

\section{TIGER geht in den Endspurt. Wie sehen die nächsten Schritte aus?}

Nach einer Verlängerung der Laufzeit über den 31. Juli 2020 hinaus, endet das Projekt TIGER nun am 28. Februar 2021. Bis dahin planen wir die Durchführung eines Workshops mit Personen, die an unserer Umfrage teilgenommen haben und bereits Erfahrungen mit der Thematik sammeln konnten.

Darüber hinaus war geplant, gemeinsam mit unseren Projektpartnern ein Symposium auf dem diesjährigen Jahreskongress der Deutschen Gesellschaft für Gerontologie und Geriatrie in Halle/Saale zu veranstalten. Dieser wurde jedoch aufgrund der Corona-Krise abgesagt.

Die Projektergebnisse werden letztlich Einfluss auf unsere Überlegungen für eine Einführung in die regelhafte Versorgung nehmen.

\section{Die Fragen stellte Daniela Goldscheck.}

\section{INFO}

Das Projekt TIGER wird vom Institut für Biomedizin des Alterns der Friedrich-Alexander-Universität Erlangen-Nürnberg (FAU) geleitet. Die praktische Umsetzung erfolgt durch geriatriespezifische Fachkräfte des Krankenhauses Barmherzige Brüder Regensburg, unterstützt vom Regensburger Ärztenetz und der AOK Bayern. Die wissenschaftliche Auswertung und Veröffentlichung erfolgt durch die FAU, das Institut für Pflegewissenschaft an der Universität Bielefeld sowie den Bundesverband Geriatrie. Über einen Zeitraum von drei Jahren wird das Projekt mit insgesamt zirka 3,7 Millionen Euro durch den Innovationsfonds des Gemeinsamen Bundesausschusses G-BA gefördert. 


\section{Update: Tagesveranstaltung Qualitätsnetzwerk Geriatrie verschoben}

Die in Ausgabe 2/2020 im Artikel „Überarbeitung des Qualitätssiegels Geriatrie geht in die heiße Phase" angekündigte Tagesveranstaltung zum Qualitätsnetzwerk Geriatrie wird auf das kommende Jahr verschoben, um ein Infektionsrisiko für die Teilnehmer auszuschließen und die Ausbreitung des Corona-Virus möglichst einzudämmen. Das haben die Mitglieder der Fachgruppe Qualität bzw. der Vorstand im Juni entschieden. „Wir sind guter Dinge, dass die Veranstaltung im ersten Quartal 2021 stattfinden kann“, sagte Dirk van den Heuvel, Geschäftsführer des BV Geriatrie. 